\title{
A study on the age related anatomical variations of some of the cerebral cortical sulci of human brain
}

\author{
Deepthi Anuraj', Anne George'2, Riya Abraham³, Neenu George ${ }^{4}$ \\ ${ }^{1,3,4}$ Assistant Professor, ${ }^{2}$ Associate Professor, Department of Anatomy, Government Medical College, Kottayam, Kerala \\ State
}

Background: The sulcal patterns may be useful in studying the mechanisms of both neuro developmental and neuro degenerative changes that occur during brain maturation and aging. Magnetic resonance imaging has facilitated the non-invasive study of human cerebral and cognitive development.Aims and Objective: To find out the age related anatomical variations of some of the cerebral cortical sulci and gyri of the brain. Materials and Methods: The study was done using the MRI images of brain of normal healthy adults aged between 2070 years. A total of 200 images were analysed consisting of that from 119 male subjects and 81 female subjects from the Department of Radiodiagnosis, Government Medical College Kottayam. Results: Using statistical analysis it was found that as the age advances there was decrease in the depth, in case of central sulcus, pre-central sulcus and post-central sulcus. The width of the pre-central sulcus and post-central sulcus was found to increase with age. The changes in the width of the central sulcus were not significant. Conclusion: The observations of the undertaken study indicated that age changes do occur in the sulci of the brain and there was a sequential change in relation to the different periods of adult life. These changes are probably due to the volume loss of the brain due to the atrophy that occurs during aging.

Key words: Sulci; Gyri; Depth and Width of sulci; MRI images; Aging
Access this article online

Website:

http://nepjol.info/index.php/AJMS

DOI: 10.3126/ajms.v12i5.33596

E-ISSN: 2091-0576

P-ISSN: 2467-9100

Copyright (c) 2021 Asian Journal of Medical Sciences

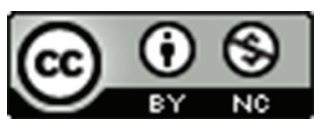

This work is licensed under a Creative Commons Attribution-NonCommercial 4.0 International License.

\section{INTRODUCTION}

The cerebrum is the largest part of the brain. It is divided into right and left cerebral hemispheres by the median longitudinal fissure. The outer layer of the cerebral hemisphere consists of gray mater called cerebral cortex. ${ }^{1}$ The mechanical process of gyral and sulcal folding occurs during brain development in gyrencephalic species, such as humans. ${ }^{2}$ This folding occurs in order to accommodate the highly extensive cerebral cortex within the rigid cranial cavity. ${ }^{3}$ The central sulcus begins by cutting the superomedial border of the hemisphere about $1 \mathrm{~cm}$ behind the midpoint between the frontal and occipital poles, runs sinuously downwards and forwards at an angle of $70^{\circ}$ and ends above the posterior ramus of the lateral sulcus. Its upper end usually extends onto the medial surface. The central sulcus forms the boundary between the motor and sensory areas of the cerebral hemisphere. This sulcus serves as a key to localize the other sulci and gyri.

The pre-central sulcus runs downwards and forwards parallel to and a little anterior to the central sulcus.The post-central sulcus runs downwards and forwards parallel to and a little posterior to the central sulcus. ${ }^{4}$ Aging is commonly defined as the accumulation of diverse deleterious changes occurring in cells and tissues with advancing age that are responsible for the increased risk of disease and death. ${ }^{5}$ Sulcal and gyral patterns reflect the effects of both maturational and neurodegenerative processes. ${ }^{6}$ The sulcal and gyral patterns reflect brain maturation processes and may indicate the effects of abnormalities in development due to either genetic or environmental factors. Based on these facts and 
observations, it was thought worthwhile to study the age changes of the major sulci and gyri of the brain.

\section{MATERIALS AND METHODS}

The radiological images for the study were obtained from the Radiodiagnosis Department Medical college Kottayam. A total of 200 MRI films of normal human brain of subjects in the age group of 20-70 years were analysed. The study subjects consisted of 119 males and 81 females. The collected images were categorized to different decades, from 20-70 years of age and the study period was 1 year from February 2012 to February 2013. Subjects with known endocrinal or psychiatric or neurological illness, head injury patients and patients with obvious pathology like swelling of brain were excluded from the study. The straight measurements were taken using software called J RULER. The sinus measurements were taken using software called GEOGEBRA. The measurements were taken using the Inverted Window method which makes the details of the sulci and gyri more obvious.

The width of the sulci sand gyri were measured at intervals of $1.5 \mathrm{~mm}$ and the average was taken. The depth and width of the central, pre-central, post-central sulci were found using axial sections. The different dimensions of the sulci and gyri obtained were tabulated and the images were photographed. The tabulation works were followed by statistical analysis. The relation between age groups and different dimensions was analysed using ANOVA test.

\section{Ethics}

The procedures followed were in accordance with the ethical standards of the Institutional ethics committee of Government Medical College Kottayam.

\section{Statistics}

All data were collected, complied and analysed using Microsoft Excel. Data analysis was done using ANOVA test. A p-value of $<0.05$ was considered to be significant

\section{RESULTS}

The mean depth of the central sulcus (in centimetres) was found to be 4.47 in the age group 20-30 years, 4.38 in the age group 31-40 years, 4.03 in the age group 41-50 years, 2.71 in the age group 51-60 years, 2.153 in the age group $>60$ years. $\mathrm{P}$ value was 0.001 .The depth was found to decrease as the age advances and was statistically significant (Table 1).

The mean depth(in centimetres)of the pre-centralsulcus was found to be 4.20 in the age group 20-30 years, 3.34 in the age group 31-40 years,3.024 in the age group 4150 years, 2.41 in the age group 51-60 years and 1.68 in the age group $>60$ years. $\mathrm{P}$ value was 0.001 which was statistically significant (Table 2).

The mean depth (in centimetres) of the post-central sulcus was found to be 2.93 in the age group 20-30 years, 2.77 in the age group 31-40 years, 2.34 in the age group $41-50$ years, 2.76 in the age group 51-60 years and 2.10 in the age group of $>60$ years. P value was 0.001 . The depth was found to decrease with increasing age except in the age group 51-60 years where there was an increase in the mean depth compared to the other age groups (Table 3).

The mean width (in centimetres) of central sulcus was found to be 0.21 in the age group 20-30 years, 0.23 in the age group 31-40 years , 0.25 in the age group $41-50$ years, 0.25 in the age group 51-60 years, 0.23 in the age group $>60$ years. There was widening of the central sulcus as age advanced. $P$ value was 0.317 . The difference was found to be non-significant (Table 4).

The mean width(in centimetres) of pre-central sulcus showed an increase with advanced age and was found to be 0.20 in the age group 20-30 years, 0.18 in the age group $31-40$ years, 0.20 in the age group of $41-50$ years, 0.21 in

\begin{tabular}{|c|c|c|c|}
\hline $\begin{array}{l}\text { Age group } \\
\text { (in years) }\end{array}$ & Mean $(\mathrm{cm})$ & $\mathbf{N}$ & Standard deviation \\
\hline $20-30$ & 4.47250 & 40 & .794422 \\
\hline $31-40$ & 4.37875 & 40 & .691042 \\
\hline $41-50$ & 4.03000 & 40 & .796161 \\
\hline $51-60$ & 2.71400 & 40 & .621659 \\
\hline$>60$ & 2.15250 & 40 & .485184 \\
\hline Total & 3.54955 & 200 & 1.162687 \\
\hline
\end{tabular}

\begin{tabular}{|c|c|c|c|}
\hline Age group (in years) & Mean $(\mathrm{cm})$ & $\mathbf{N}$ & Standard Deviation \\
\hline $20-30$ & 4.20200 & 40 & 619943 \\
\hline $31-40$ & 3.33650 & 40 & .572491 \\
\hline $41-50$ & 3.02425 & 40 & .544341 \\
\hline $51-60$ & 2.40775 & 40 & .542381 \\
\hline$>60$ & 1.67675 & 40 & .502187 \\
\hline Total & 2.92945 & 200 & 1.017571 \\
\hline
\end{tabular}

\begin{tabular}{|c|c|c|c|}
\hline Age group (in years) & Mean $(\mathrm{cm})$ & $\mathbf{N}$ & Standard deviation \\
\hline $20-30$ & 2.92625 & 40 & .406118 \\
\hline $31-40$ & 2.76500 & 40 & .692046 \\
\hline $41-50$ & 2.34075 & 40 & .545360 \\
\hline $51-60$ & 2.76275 & 40 & .687728 \\
\hline$>60$ & 2.10375 & 40 & .416861 \\
\hline Total & 2.57970 & 200 & .637187 \\
\hline
\end{tabular}


the age group 51-60 years, 0.27 in the age group of $>60$ years. P value was .001. The difference was found to be statistically significant (Table 5).

In 20-30 years of age, the mean width (in centimetres) of post-central sulcus was found to be 0.21 in the age group, 0.36 in the age group of $31-40$ years, 0.25 in the age group 41-50 years, 0.24 .in the age group 51-60 years, 0.46 in the age group $>60$ years. The difference was found to be statistically significant with a P value of 0.001 (Table 6).

In 20-30 years of age, the mean width (in centimetres) of post-central sulcus was found to be 0.21 in the age group, 0.36 in the age group of $31-40$ years, 0.25 in the age group $41-50$ years, 0.24 .in the age group 51-60 years, 0.46 in the age group $>60$ years. The difference was found to be statistically significant with a $\mathrm{P}$ value of 0.001 (Table 7). Histogram shown in Figure 1 and 2 shows width and depth of sulci in different age groups of the study subjects.

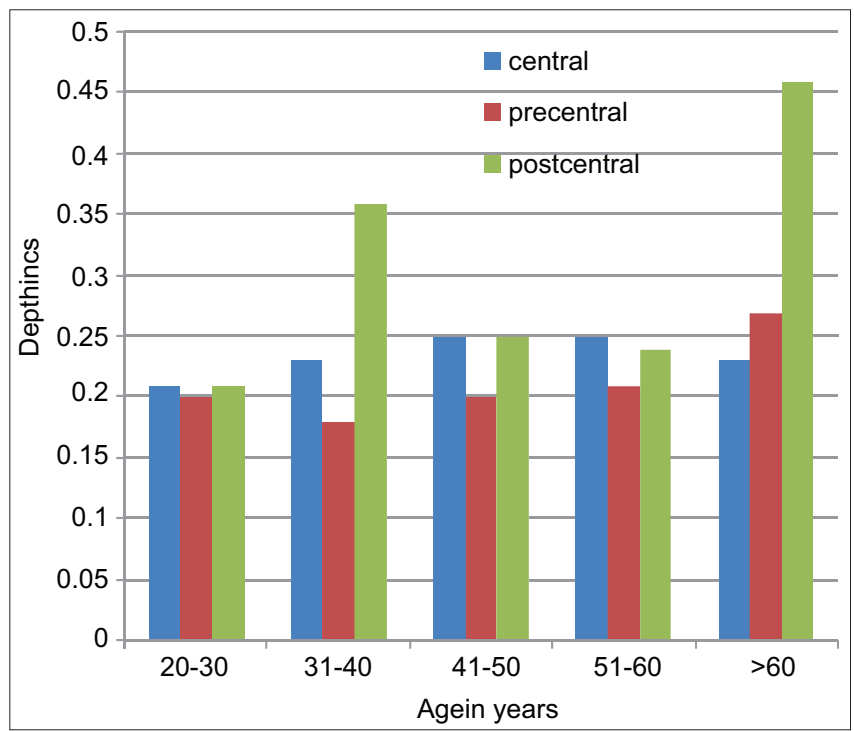

Figure 1: Histogram showing depth of the sulci in different age groups

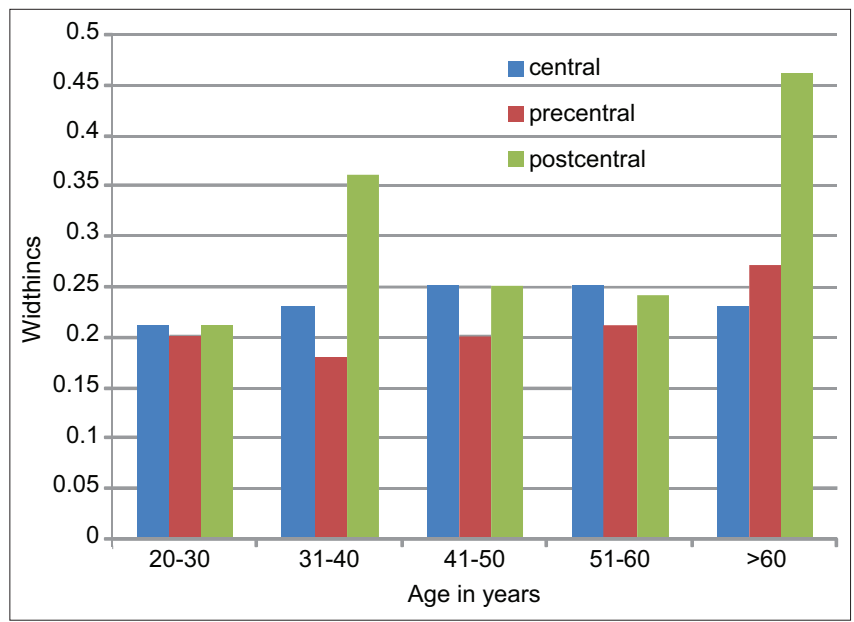

Figure 2: Histogram showing width of the sulci in different age groups
The MRI shows the central, pre-central and post-central sulci, in 20-30 years of age (Image 1) in 31-40 years of age (Image 2), in 41-50 years of age (Image 3), in 51-60 years of age (Image 4 ) and $>60$ years of age is shown in Image 5 . The MRI inverted window axial section showing the central sulcus is presented in Image 6. Image 7 shows MRI inverted window axial section showing the pre-central and post-central sulci.

\section{DISCUSSION}

The purpose of the study undertaken was to demonstrate the age related radiological changes in the major sulci and gyri of the human brain. The observations recorded demonstrated that changes do occur in the dimensions of the sulci and gyri of the brain as age advances.

\begin{tabular}{|c|c|c|c|}
\hline Age group (in years) & Mean $(\mathrm{cm})$ & $\mathbf{N}$ & Standard deviation \\
\hline $20-30$ & .21048 & 40 & .099061 \\
\hline $31-40$ & .23090 & 40 & .087271 \\
\hline $41-50$ & .25082 & 40 & .107774 \\
\hline $51-60$ & .25080 & 40 & .107805 \\
\hline$>60$ & .22980 & 40 & .086572 \\
\hline Total & .23456 & 200 & .098330 \\
\hline
\end{tabular}

\begin{tabular}{|c|c|c|c|}
\hline Age group (in years) & Mean $(\mathrm{cm})$ & $\mathbf{N}$ & Standard deviation \\
\hline $20-30$ & .20400 & 40 & .128288 \\
\hline $31-40$ & .18228 & 40 & .062662 \\
\hline $41-50$ & .20498 & 40 & .064885 \\
\hline $51-60$ & .21310 & 40 & .063430 \\
\hline$>60$ & .26540 & 40 & .068172 \\
\hline Total & .21395 & 200 & .085378 \\
\hline
\end{tabular}

$\begin{aligned} & \text { Table 6: The width of the post-central sulcus in } \\
& \text { relation to different age groups }\end{aligned}$
\begin{tabular}{lccc}
\hline Age group(in years) & Mean (cm) & $\mathbf{N}$ & Standard deviation \\
\hline $20-30$ & .21418 & 40 & .064027 \\
$31-40$ & .36400 & 40 & .554736 \\
$41-50$ & .25382 & 40 & .064137 \\
$51-60$ & .24475 & 40 & .064881 \\
$>60$ & .46040 & 40 & .073172 \\
Total & .30743 & 200 & .268803 \\
\hline
\end{tabular}

Table 7: The width of the post-central sulcus in
relation to different age groups
\begin{tabular}{lccc} 
Age group (in years) & Mean (cm) & $\mathbf{N}$ & Standard Deviation \\
\hline $20-30$ & .21418 & 40 & .064027 \\
$31-40$ & .36400 & 40 & .554736 \\
$41-50$ & .25382 & 40 & .064137 \\
$51-60$ & .24475 & 40 & .064881 \\
$>60$ & .46040 & 40 & .073172 \\
Total & .30743 & 200 & .268803 \\
\hline
\end{tabular}




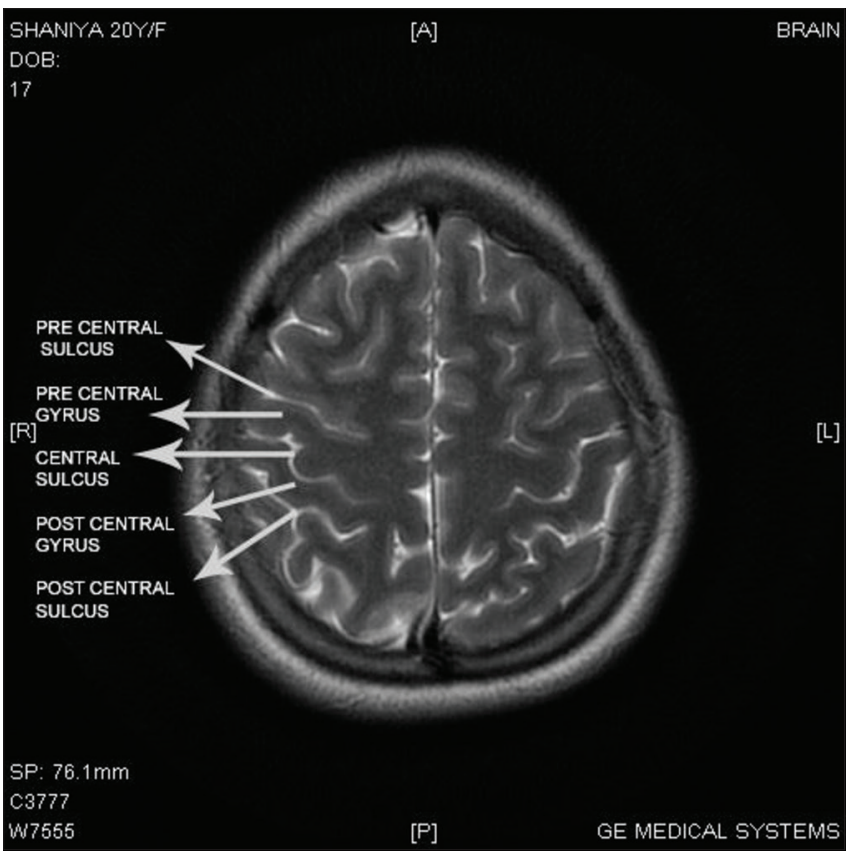

Image 1: MRI showing the central, precentral and post-central sulci, in 20-30 years of age

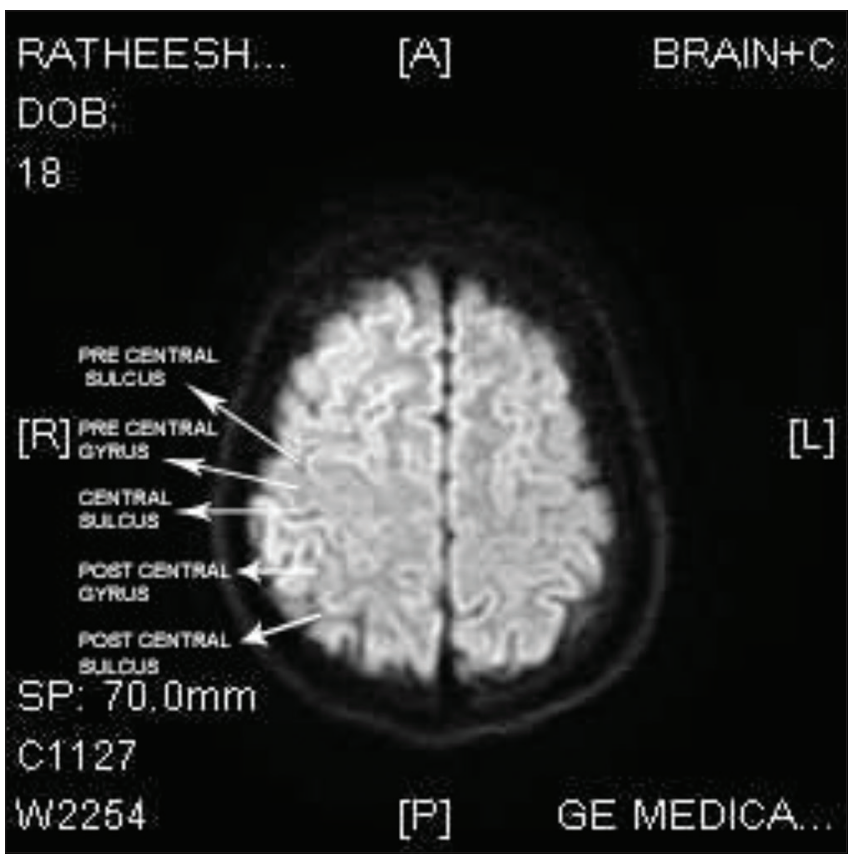

Image 2: MRI showing the central, pre-central and post-central sulci in 31-40 years of age

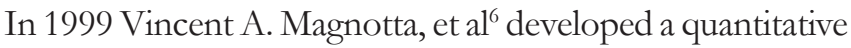
automated method to achieve in vivo measurement of sulcal and gyral characteristics. This was the first study to examine the age related changes in surface characteristics of the cerebral cortex by using quantitative measures of sulcal and gyral shape.

They found that atrophic gyri have a diminution in their rounded curvature and become more sharply and

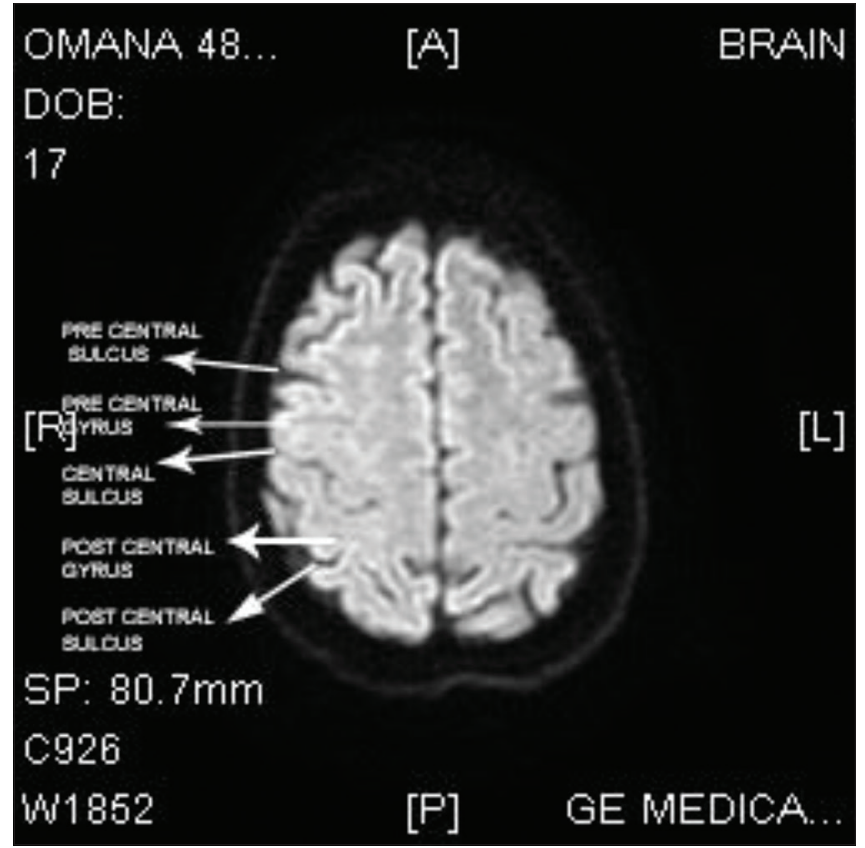

Image 3: MRI showing the central, pre-central and post-central sulci, in $41-50$ years of age

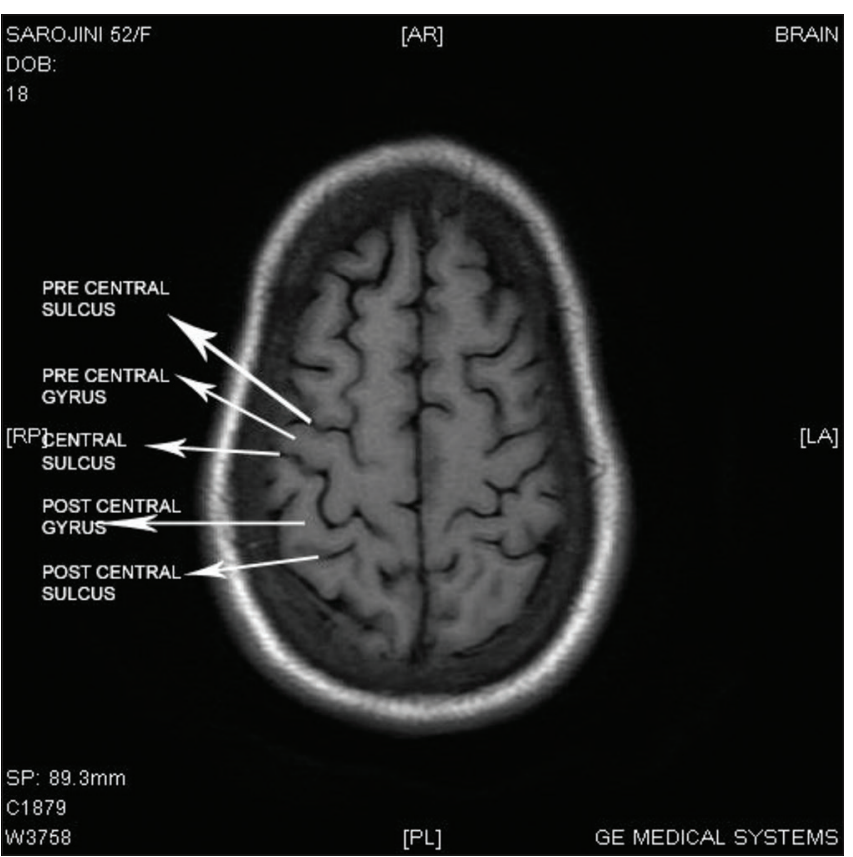

Image 4: MRI showing the central, pre-central and post-central sulci in $51-60$ years of age

steeply curved, giving them a larger (more positive) gyral curvature index. Atrophic sulci, on the other hand, become less curved and more flattened, producing a larger (less negative) sulcal curvature index. These two indices can be considered to provide a quantitative index of the degree of atrophy. ${ }^{6}$

In a cross-sectional study by Coffey C. E, W. E. Wilkinson et al, (1992) seventy-six healthy adults underwent magnetic 


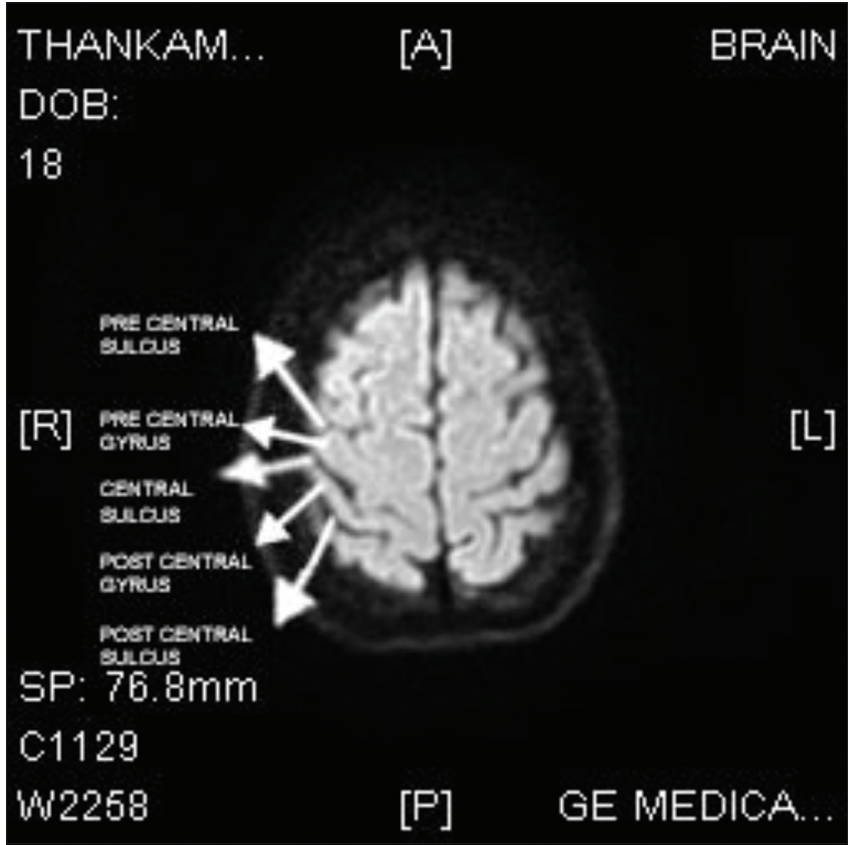

Image 5: MRI showing the central, pre-central and post-central sulci in $>60$ years of age

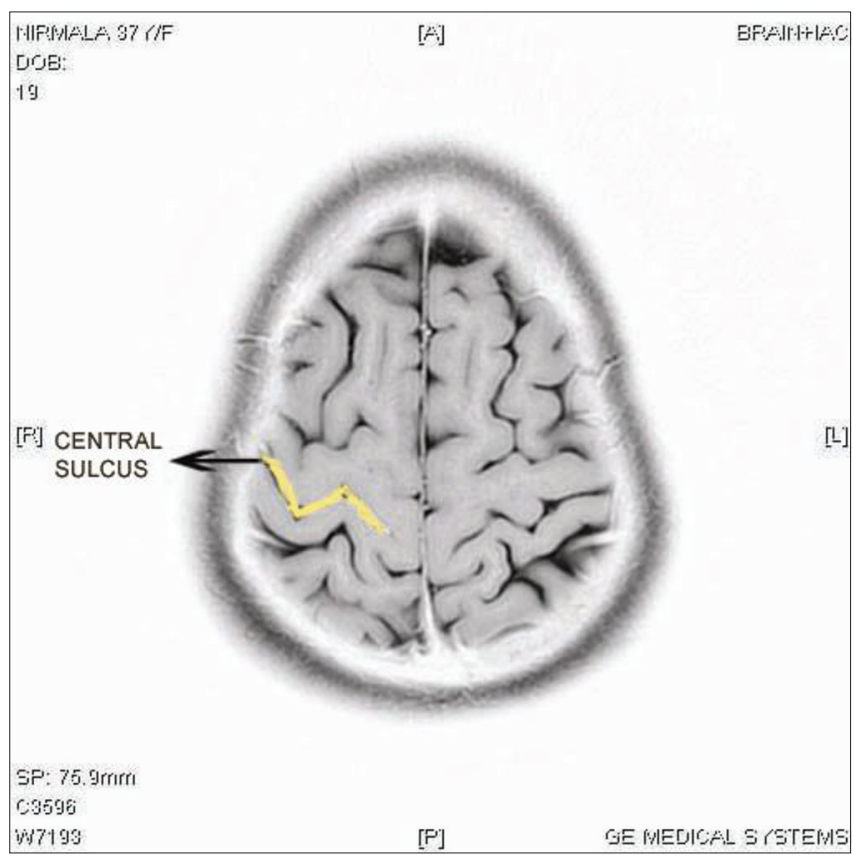

Image 6: MRI Inverted window axial section showing the central sulcus

resonance imaging $(1.5 \mathrm{~T})$ to investigate the effects of age on regional cerebral volumes and on the frequency and severity of cortical atrophy, lateral ventricular enlargement, and subcortical hyperintensity. Increasing age was associated with decreasing volumes of the cerebral hemisphere.

Alonso I et al (2010) had measured the depth of the Insula and Sylvian fissure in a standard transventricular axial

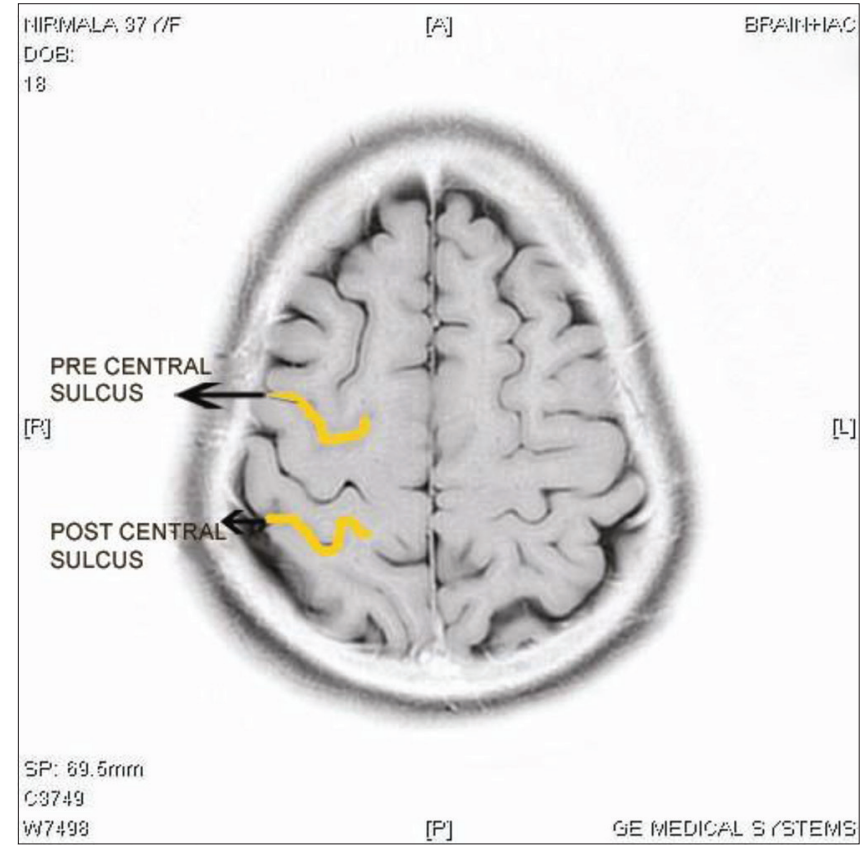

Image 7: MRI Inverted window axial section showing the pre-central and Post-central sulci

plane of the fetal head; the depth of the Parieto-occipital in a plane above and parallel to that used for the insula; and the depth of the Calcarine fissure in a coronal view of the posterior fossa. The depth of these four structures increased with increasing gestational age. ${ }^{8}$

Quantitative measurements of sulci and gyri can potentially provide important information concerning normal brain development and aging as well as a variety of disease processes. It may also be useful in studying the mechanisms of both neuro developmental and neuro degenerative changes that occur during brain maturation and aging. ${ }^{6}$

In the present study, it was found that in case of depth of the sulci, there was decrease in the depth as the age advanced; in case of central, pre-central and post-central sulci. These changes were statistically significant. In case of width of the sulci there was increase in the width of the pre-central and post-central sulci and these changes were statistically significant. There was increase in the width of the central sulcus too, but that was not statistically significant.

The sulcal and gyral patterns reflect brain maturational processes and may indicate the effects of abnormalities in development due to either genetic or environmental factors.

The observations of the undertaken study indicate that age changes do occur in the sulci and gyri of the brain and there is a definite pattern of sequential changes in relation to the different periods of adult life. These changes are due 
to the volume loss of the brain due to the cerebral atrophy that occurs during aging. ${ }^{9}$

\section{CONCLUSION}

Enhanced interest in diseases and disabilities associated with aging has led to many studies dealing with various changes during the aging process, hoping that methods will be found for delaying the onset, lessening the severity or perhaps preventing some of the pathological changes of aging. In our study, it was found that in case of depth of the sulci, there was decrease in the depth of central, precentral and post-central sulci as the age advanced. There was increase in the width of the central, pre-central and post-central sulci, as the age advanced

\section{Limitations of the study}

The number of study samples can be increased and further studies can be conducted to find out the relation between gender difference and changes of the cerebral cortical sulci and gyri

\section{ACKNOWLEDGMENTS}

The authors are grateful to the Department of Anatomy, Medical college, Kottayam

\section{REFERENCES}

1. Vishram Singh. Text Book of Anatomy, Head, Neck and Brain .3 $3^{\text {rd }}$ edn. page 385.

2. Welker WI. Cerebral Cortex. 1990; 8B: 134. http://doi-org 443. webvpn. fjmu. edu.cn/10.1007/978-1-4615-3824-0_1

3. Cerebrum-Text book of Clinical Neuro Anatomy 2nd edn Page183.

4. URL:https://neupsykey.com/cerebrum/ Page12.

5. Harman D. The biologic clock: the mitochondria. J Am Geriatr Soc. $1972 ; 20: 145-147$.

https://doi.org/10.1111/j.1532-5415.1972.tb00787.x

6. Vincent A. Magnotta and Nancy C. Andreasen and Susan K. Schultz. Quantitative In Vivo Measurement of Gyrification in the Human Brain: Changes Associated with Aging Cereb Cortex.1999; 9 (2): 151-160.

https://doi.org/10.1093/cercor/9.2.151

7. Coffey CE, Wilkinson WE and Parashos LA. Quantitative cerebral anatomy of the aging human brain.A cross-sectional study using magnetic resonance imaging Neurology.1992; 42(3):527.

https://doi.org/10.1212/WNL.42.3.527

8. Alonso I, Borenstein M, Grant G, Narbona I and Azumendi G. Depth of brain fissures in normal fetuses by prenatal ultrasound between 19 and 30 weeks of gestation. Ultrasound Obstet Gynecol.2010;36(6):693-699. https://doi.org/10.1002/uog.7660

9. Jin K, Zhan $T$ and Shaw M. Relationship Between Sulcal Characteristics and Brain Aging.2018; 10:339. https://doi.org/10.3389/fnagi.2018.00339

\footnotetext{
Authors' contributions:

DA-Concept and design of the study, statistical analysis and interpretation of results, manuscript preparation; AG-Co-ordination of the overall study, manuscript editing; RA-Reviewed the manuscript preparation; NG-Critical revision of the manuscript.

Work attributed to:

Government Medical College Kottayam.

Orcid ID:

Dr. Deepthi Anuraj- (D) https://orcid.org/0000-0002-2737-1253

Dr. Anne George- (10) https://orcid.org/0000-0001-8055-4529

Sources of Support: None, Conflict of Interests: None.
} 